\title{
Familias de agua subterránea y distribución de sólidos totales disueltos en el acuífero de La Paz Baja California Sur, México
}

\author{
Groundwater types and total dissolved solids distribution in the aquifer \\ of La Paz Baja California Sur, México
}

\section{Arturo Cruz Falcón ${ }^{1 *}$, Enrique Troyo Diéguez ${ }^{1}$, Janette M. Murillo Jiménez², José Luis García Hernández ${ }^{3}$ y Bernardo Murillo Amador ${ }^{1}$}

\footnotetext{
${ }^{1}$ Centro de Investigaciones Biológicas del Noroeste (CIBNOR). Av. Instituto Politécnico Nacional 195, Col. Playa Palo de Sta. Rita Sur. 23096 La Paz, B.C.S., México.

*Autor responsable (afalcon04@cibnor.mx)

${ }^{2}$ Centro Interdisciplinario de Ciencias Marinas (CICIMAR)-IPN. Av. Instituto Politécnico Nacional s/n, Col. Playa Palo de Sta. Rita Sur. Apdo. Postal 592. 23096 La Paz, B.C.S., México.

${ }^{3}$ Universidad Juárez del Estado de Durango (UJED). Constitución 404, Centro. 34100 Durango, Dgo., México.
}

\section{RESUMEN}

El acuífero de La Paz se encuentra desde hace varios años en condición de sobreexplotación, lo que ha causado problemas de contaminación por intrusión marina. En 2013 se conformó una red de monitoreo de pozos, que incluyó el uso públicourbano, agrícola, pecuario y de servicios, donde se realizaron muestreos representativos de la temporada de estiaje y lluvias. Se midió la concentración de sólidos totales disueltos (STD), temperatura, oxígeno disuelto y $\mathrm{pH}$. Los resultados mostraron que la mayoría de los pozos agrícolas contenían alta concentración de STD a diferencia de los pozos de agua potable con concentración inferior a $1000 \mathrm{mg} \mathrm{L}^{-1}$, que es el límite permisible por la norma oficial mexicana (NOM). También se analizaron datos de iones mayoritarios de 14 pozos cercanos a la costa con alta concentración de STD, con los que se elaboró un diagrama de Piper, encontrándose dos tipos o familias principales de agua: clorurada-cálcica y clorurada-sódica. La presencia de sodio en concentraciones que superan los $200 \mathrm{mg} \mathrm{L}^{-1}$ en 8 pozos, y de magnesio con más de $50 \mathrm{mg} \mathrm{L}^{-1}$ en 10 pozos, sobrepasaron la NOM y se consideró que su origen es marino. La concentración de calcio mayor a los $250 \mathrm{mg} \mathrm{L}^{-1}$ en 7 pozos, supone la existencia de capas de sedimentos de origen químico-orgánico en la estratigrafía interior del acuífero, a través de las cuales circula el agua subterránea. Los cloruros

Cita recomendada:

Cruz Falcón, A., E. Troyo Diéguez, J. M. Murillo Jiménez, J. L. García Hernández y B. Murillo Amador. 2017. Familias de agua subterránea y distribución de sólidos totales disueltos en el acuífero de La Paz, Baja California Sur, México. Terra Latinoamericana 36: 39-48.

DOI: https://doi.org/10.28940/terra.v36i1.316 en concentraciones superiores a los $1000 \mathrm{mg} \mathrm{L}^{-1}$ en 6 pozos con elevada concentración de STD, sugiere que es producto de la intrusión marina. Con base en la distribución de la concentración de STD se delimitaron dos áreas amplias afectadas por la intrusión marina, y junto con la distribución de las familias de agua, se identificaron tres zonas principales del acuífero.

Palabras clave: intrusión marina, calidad del agua subterránea, contaminación por sales, contaminación de agua subterránea en zonas costeras.

\section{SUMMARY}

The aquifer of $\mathrm{La} \mathrm{Paz}$ has been overexploited for several years, causing contamination by seawater intrusion. A well-monitoring network, which included public-urban, agricultural, livestock and service use water wells, was set up in 2013. Representative sampling during the dry and rainy seasons was carried out. Concentration of total dissolved solids (TDS), temperature, dissolved oxygen and $\mathrm{pH}$ were measured. Results indicate that most agricultural wells contain high TDS concentration, compared to potable water wells with concentration below $1000 \mathrm{mg} \mathrm{L}^{-1}$, which is the limit allowed by the Mexican official standard (MOS). Majority ion data from 14 wells near the coast with high concentration of TDS were also analyzed, with which a Piper diagram was 
constructed. Two main types of water were found: calcium-chloride and sodium-chloride. The presence of sodium in concentrations exceeding $200 \mathrm{mg} \mathrm{L}^{-1}$ in 8 wells, and magnesium with more than $50 \mathrm{mg} \mathrm{L}^{-1}$ in 10 wells, both above the MOS, reveal that its origin is seawater. Calcium concentrations above $250 \mathrm{mg} \mathrm{L}^{-1}$ in 7 wells points to the existence of sedimentary layers of chemical-organic origin that compose the inner stratigraphy of the aquifer through which groundwater circulates. High chloride concentrations of more than $1000 \mathrm{mg} \mathrm{L}^{-1}$ in 6 wells with high concentration of TDS, suggests that its source is seawater intrusion. Based on the distribution of TDS concentration, two broad areas affected by seawater intrusion were delimited, and along with the distribution of water families, three main aquifer zones were identified.

Index words: seawater intrusion, groundwater quality, salts contamination, contamination of groundwater in coastal areas.

\section{INTRODUCCIÓN}

Actualmente existe un problema serio de escasez de agua, contaminación y deterioro de los acuíferos que abastecen agua a las poblaciones (Arreguín Cortés, 2015) ${ }^{1}$. El acuífero de La Paz no es la excepción y presenta sus propias dificultades.

Durante los últimos años, este acuífero se ha visto notablemente afectado por la sobreexplotación debido a la extracción de mayores volúmenes de agua con respecto a la recarga natural debido a las lluvias, lo cual ha provocado contaminación por intrusión marina por tratarse de un acuífero costero (CNA, 1997; CIGSA, 2001; Cruz Falcón et al., 2013). Los problemas van en aumento, ya que se observa un incremento cada vez mayor de la profundidad de hasta $10 \mathrm{~m}$, al nivel del agua subterránea en áreas agrícolas irrigadas donde no se controla la extracción de la misma (Tamez Meléndez et al., 2016). La recarga estimada en varios estudios es de 20 a $23 \mathrm{Mm}^{3}$, con una descarga entre 29 a $32 \mathrm{Mm}^{3}$, que resulta en un balance cercano a los $-10 \mathrm{Mm}^{\mathrm{año}} \mathrm{o}^{-1}$ (Cruz Falcón et al., 2013). Esto a su vez originado por el aumento en la demanda debido al crecimiento de la población de la ciudad de La Paz a una tasa anual promedio del 5\% (Rivera Rosas, 2012)². Así mismo, la disponibilidad de agua para los pobladores ha disminuido de forma notable, un $24 \%$ del 2000 al 2010, y del 2010 al 2020 se estima en más del 40\%, debido a que no existen otras fuentes de abastecimiento, excepto lo que aporta el mismo acuífero, razón por la cual se ha visto afectada la calidad del agua (CONAGUA, 2010).

En el estado de Baja California Sur, el abasto de agua para la población depende casi en su totalidad del agua de los acuíferos y su escasez ha sido el factor limitante para el desarrollo en la región y de La Paz (CCA, CONAGUA, FMEA, 2005), por lo que el mayor reto es la provisión de agua para sus residentes, en suficiente cantidad y calidad (Steinitz et al., 2006).

La composición química de las aguas subterráneas es muy variable, depende de la composición natural de rocas y sedimentos, lixiviación de vertederos, residuos industriales, fugas de aguas residuales, suelos con fertilizantes y pesticidas en áreas agrícolas y residuos de minas potencialmente enriquecidos con elementos tóxicos (Carrillo Chávez, 1996) ${ }^{3}$. Por lo tanto, el agua contenida en los acuíferos es producto del agua de lluvia que se infiltra y fluye a través del suelo y las rocas llevando consigo minerales y elementos naturales, así como productos químicos o contaminantes. En los acuíferos costeros es común el incremento de sales de sodio y magnesio a causa de la intrusión marina.

La evolución hidrogeoquímica del agua subterránea se puede definir con base en información de los sólidos totales disueltos (STD) (Carrillo Chávez et al., 2000). Los cuales son una medida de la cantidad de materia, sustancias, elementos y sales disueltas en el agua. Comprenden las sales inorgánicas, principalmente de calcio, magnesio, potasio y sodio, bicarbonatos, cloruros y sulfatos, y pequeñas cantidades de materia orgánica que están disueltas en el agua. El límite permisible de STD en el agua potable definido en la Norma Oficial Mexicana (NOM-127-SSA1-1994) es de $1000 \mathrm{mg} \mathrm{L}^{-1}$.

El propósito de este estudio fue la valoración del acuífero de La Paz para el año 2013, en relación con la distribución de STD y sales que definen la intrusión de agua de mar, así como otros procesos que afectan la calidad del agua subterránea. El acuífero presenta

\footnotetext{
${ }^{1}$ Arreguín Cortés, F. 2015. Los retos de la investigación y la transferencia de tecnología para la mejor gestión de las aguas subterráneas. Conferencia Magistral. II Coloquio Nacional de Agua Subterránea en México. Senado de la República del 11-13 de noviembre de 2015. México, D. F.

${ }^{2}$ Rivera Rosas, J. 2012. Efectos socioambientales en la cuenca costera de La Paz, BCS., México, inducidos por el cambio climático. Tesis de Maestría. UABCS. La Paz, BCS, México.135 p.

${ }^{3}$ Carrillo Chavez, A. 1996. Environmental geochemistry of the San Antonio-El Triunfo mining area, southernmost Baja California Peninsula Mexico. Ph. D. Thesis. Department of Geology and Geophysics, University of Wyoming, Laramie, Wyoming USA, 186 p.
} 
efectos de la intrusión marina inferidos por las altas concentraciones de STD, cloruros, sodio y magnesio, que rebasan los límites establecidos en la NOM.

\section{Área de Estudio}

El acuífero de La Paz se asienta en el subsuelo del área de la ciudad de La Paz, localizada en la parte sur de la península de Baja California, México (Figura 1). Se clasifica como un acuífero libre (CIGSA, 2001; Escolero y Torres Onofre, 2007; CONAGUA, 2009) compuesto principalmente por materiales aluviales de alta permeabilidad que suprayacen a sedimentos arcillo arenosos, rocas volcánicas fracturadas y piroclastos de baja permeabilidad. La base de estas unidades corresponde a un complejo ígneo metamórfico de naturaleza impermeable (CNA, 1997). Este acuífero se define también como un acuífero costero porque su frontera norte limita con el mar, situación que lo hace vulnerable a la contaminación por intrusión marina (Cruz Falcón et al., 2013). El acuífero se distribuye en toda la zona del valle cubierta por depósitos aluviales dentro de la cuenca y se extiende sobre una superficie de aproximadamente $858 \mathrm{~km}^{2}$, sin embargo, la zona de explotación se concentra en un área de alrededor de 200 km² (Cruz Falcón et al., 2013).

El acuífero de La Paz es uno de los más importantes de la entidad, ya que abastece a la ciudad de La Paz (capital del estado), la cual demanda aproximadamente dos terceras partes del agua subterránea que se extrae,

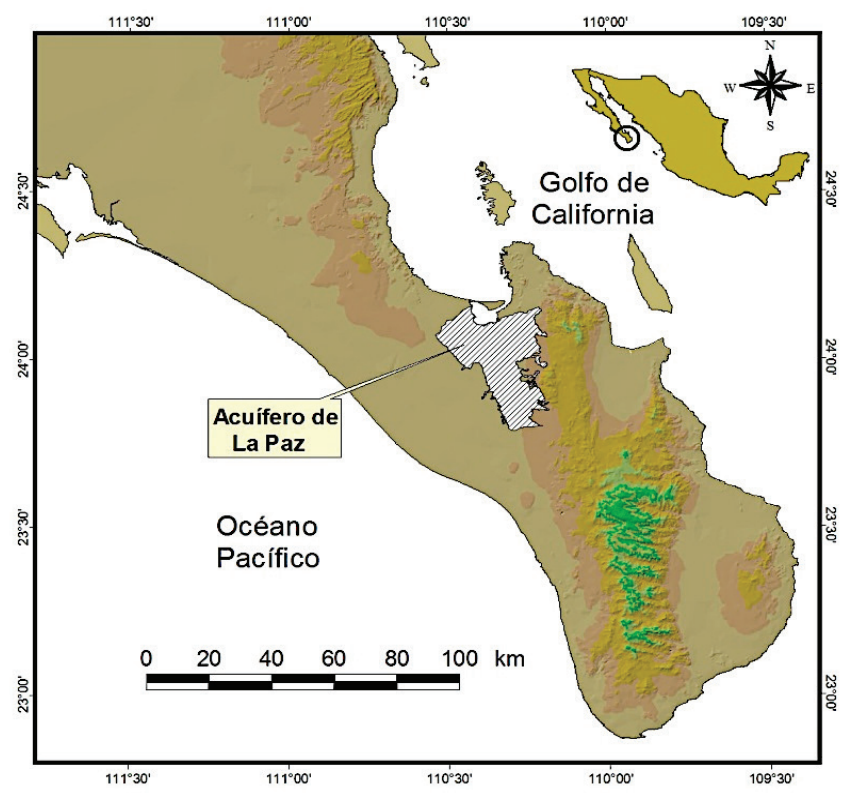

Figura 1. Localización del acuífero de La Paz. también abastece a una zona agrícola importante. Actualmente el acuífero está sobre concesionado y se encuentra en condiciones de sobreexplotación debido a que las extracciones anuales han sobrepasado la disponibilidad total del agua, ya que algunos usuarios entre ellos el Organismo Operador Municipal del Sistema de Agua Potable, Alcantarillado y Saneamiento (OOMSAPAS) de La Paz utiliza volúmenes mayores a los de la dotación por habitante (CONAGUA, 2009), posiblemente debido a la enorme cantidad de fugas en la red de distribución y desperdicio en los domicilios y negocios por falta de medidores.

\section{MATERIALES Y MÉTODOS}

El estudio se llevó a cabo en el año 2013. A partir del trabajo de prospección en el área de estudio y de las características de los pozos, se obtuvo un mapa base para llevar a cabo el muestreo (Figura 2). Este mapa incluye un total de 61 pozos, 30 de agua potable, 28 de uso agrícola, dos de uso pecuario y uno de servicios. Se realizaron dos campañas de muestreo a lo largo del año, durante la estación seca (marzo-abril) y después de la estación húmeda (noviembre). Se hicieron las mediciones de sólidos totales disueltos (STD), en agua de 60 pozos, con el medidor Multiparámetro Hanna Modelo MPS-YSI556, calibrado previamente en el Laboratorio de Análisis Químico de Agua del Centro de Investigaciones Biológicas del Noroeste (CIBNOR).

Con datos de 14 pozos muestreados y analizados

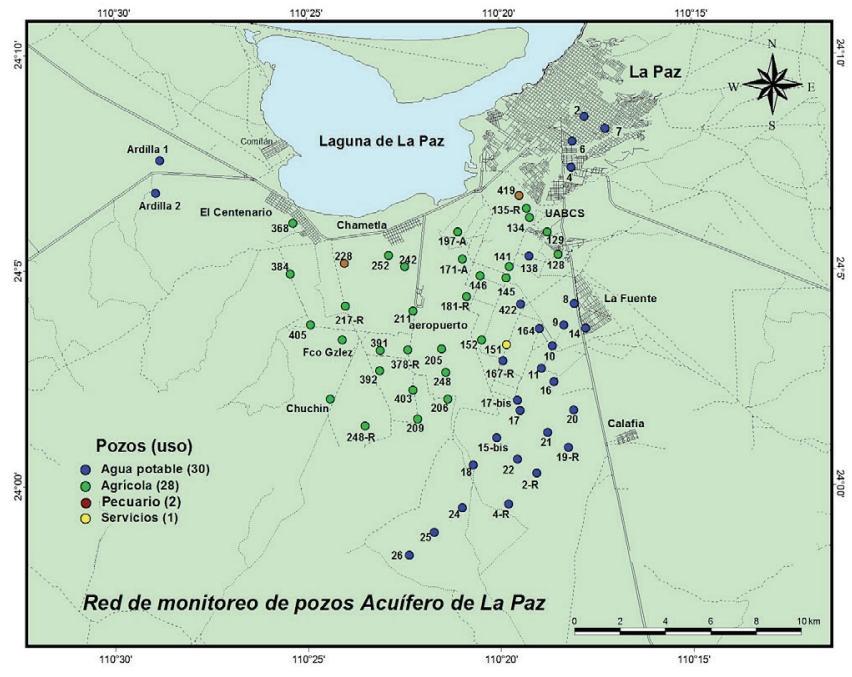

Figura 2. Mapa base de la red de monitoreo de pozos del acuífero de La Paz. Se incluyen 30 pozos de agua potable, 28 agrícolas, dos pecuarios y uno de servicios. 
por la Comisión Nacional del Agua (CONAGUA) para los años 2008, 2009 y 2013, se elaboró un diagrama de Piper para determinar las familias de agua predominantes utilizando las concentraciones de los iones principales (Freeze y Cherry, 1979). Se generaron mapas de distribución de la concentración de las variables medidas y analizadas con el programa ArcGIS (ESRI, 2009) del Environmental Systems Research Institute (ESRI) para sistemas de información geográfica, en proyección geográfica con referencia al Datum WGS84.

Solamente se analizaron los datos de STD, así como los datos de aniones y cationes principales de 14 pozos obtenidos de la CONAGUA.

\section{RESULTADOS Y DISCUSIÓN}

Es necesario establecer que los resultados de las diferencias entre los tiempos de muestreo de agua de pozo son representativos de lo que ocurre antes y después de la temporada de lluvias para el año 2013, más no del comportamiento general del acuífero. Para esto se requiere de un periodo de monitoreo más largo, al menos de 3 a 5 años.

\section{Distribución de Sólidos Totales Disueltos (STD)}

La mínima concentración de STD medida fue de $350 \mathrm{mg} \mathrm{L}^{-1}$ en el pozo 4-R, mientras que la máxima de $6200 \mathrm{mg} \mathrm{L}^{-1}$ en el pozo 217-R (Figura 2). Entre la temporada de estiaje y lluvias la concentración de STD, en un mismo pozo, se incrementó hasta $554 \mathrm{mg} \mathrm{L}^{-1}, \mathrm{y}$ disminuyó hasta $264 \mathrm{mg} \mathrm{L}^{-1}$. Se observó un incremento de STD después de la temporada de lluvias en un 53\% de los pozos, principalmente en la zona cercana a la línea costera, no así en la zona central donde se ubica la batería de pozos de agua potable, donde los valores se mantuvieron o disminuyeron.

De los 61 pozos muestreados (Figura 2), 27 pozos presentaron concentraciones de STD por arriba de los $1000 \mathrm{mg} \mathrm{L}^{-1}$, de estos, 11 pozos con 1000 a $2000 \mathrm{mg} \mathrm{L}^{-1}$ (Ardilla 2, 6, 10 y 164 de agua potable; 134, 164, 378-R, 384, 392 y 403 agrícolas; y 151 de servicios); 8 pozos con 2000 a $3000 \mathrm{mg} \mathrm{L}^{-1}(138$ y 422 de uso agrícola-agua potable; Fco. González, 135-R, 145, 211 y 368 agrícolas; y 228 pecuario); 7 pozos entre 3000 a $4000 \mathrm{mg} \mathrm{L}^{-1}(141,146,242,252,391$ y 419 agrícolas; y 419 pecuario); y el pozo 217-R con más de $6000 \mathrm{mg} \mathrm{L}^{-1}$. Los 34 pozos restantes presentaron concentraciones de STD por debajo de los $1000 \mathrm{mg} \mathrm{L}^{-1}$ (Ardilla 2, 2, 2-R, 4, 4-R, 7, 8, 11, 9, 14, 15-Bis, 16, 17, 17-Bis, 18, 19-R, 20, 21, 22, 24, 25, 26 y 167-R de agua potable; y Chuchín, 152, 197-A, 181-R, 205, 206, 209, 248, 248-R y 405 de uso agrícola).

En general la concentración de STD en los pozos de agua disminuyó de la costa hacia el interior del acuífero. Se delimitaron dos zonas con alta concentración de STD que están relacionadas con la intrusión marina y el excesivo bombeo de pozos principalmente agrícolas. Para esto se consideró como límite interno de la intrusión marina el contorno de $2000 \mathrm{mg} \mathrm{L}^{-1}$ de STD. Una zona se extiende aproximadamente $6.25 \mathrm{~km}$ de la costa, hacia el sur, entre los ejidos Chametla y El Centenario, y la otra de aproximadamente $5.5 \mathrm{~km}$ de la costa, al este del ejido Chametla y sur de la ciudad de La Paz (Figuras 3a y 3b).

Los pozos agrícolas y pecuarios 134, 146, 228, 252, 368 y 419 con concentración de STD entre 1900 y $3500 \mathrm{mg} \mathrm{L}^{-1}$, son los más cercanos a la línea de costa y tuvieron el incremento más significativo, entre la temporada de secas y húmeda, de 200 a $550 \mathrm{mg} \mathrm{L}^{-1}$, probablemente a causa de la misma intrusión marina. El pozo agrícola 209 con concentración de STD por debajo de la NOM, presentó una disminución de $131 \mathrm{mg} \mathrm{L}^{-1}$, y el pozo agrícola 135-R con concentración de STD por arriba de la NOM, disminuyó $264 \mathrm{mg} \mathrm{L}^{-1}$, entre la temporada de secas y lluvias, debido a una reducción en el bombeo. En el resto de los pozos, ubicados principalmente en la parte central y sur del acuífero, la concentración de STD se incrementó hasta $39 \mathrm{mg} \mathrm{L}^{-1}$, se redujo hasta $64 \mathrm{mg} \mathrm{L}^{-1}$, o se mantuvo igual (Figuras $3 \mathrm{a}$ y $3 \mathrm{~b}$ ). En esta zona central y sur del acuífero con concentraciones de STD menores de $1000 \mathrm{mg} \mathrm{L}^{-1}$, probablemente se deba a una corta estancia del agua dulce como resultado de un flujo rápido. Esta característica supone que existe una buena permeabilidad del sedimento donde se encuentra la mayoría de los pozos de agua potable, pero también debido al material de baja permeabilidad presente en la línea costera, lo que limita la intrusión marina, incluso cuando los niveles de agua subterránea están por debajo del nivel del mar (CNA, 1997). En esta zona la conductividad hidráulica fluctúa entre 29.49 y $51.78 \mathrm{~m} \mathrm{día}^{-1}$, superior a las áreas adyacentes (CIGSA 2001; Dragoo y Maddock, 2004). De los 23 pozos ubicados aquí, los cuales suministran agua a la ciudad de La Paz, tres de ellos (138, 164 y 422) (Figuras 3a y $3 b)$ presentaron una concentración de STD por 


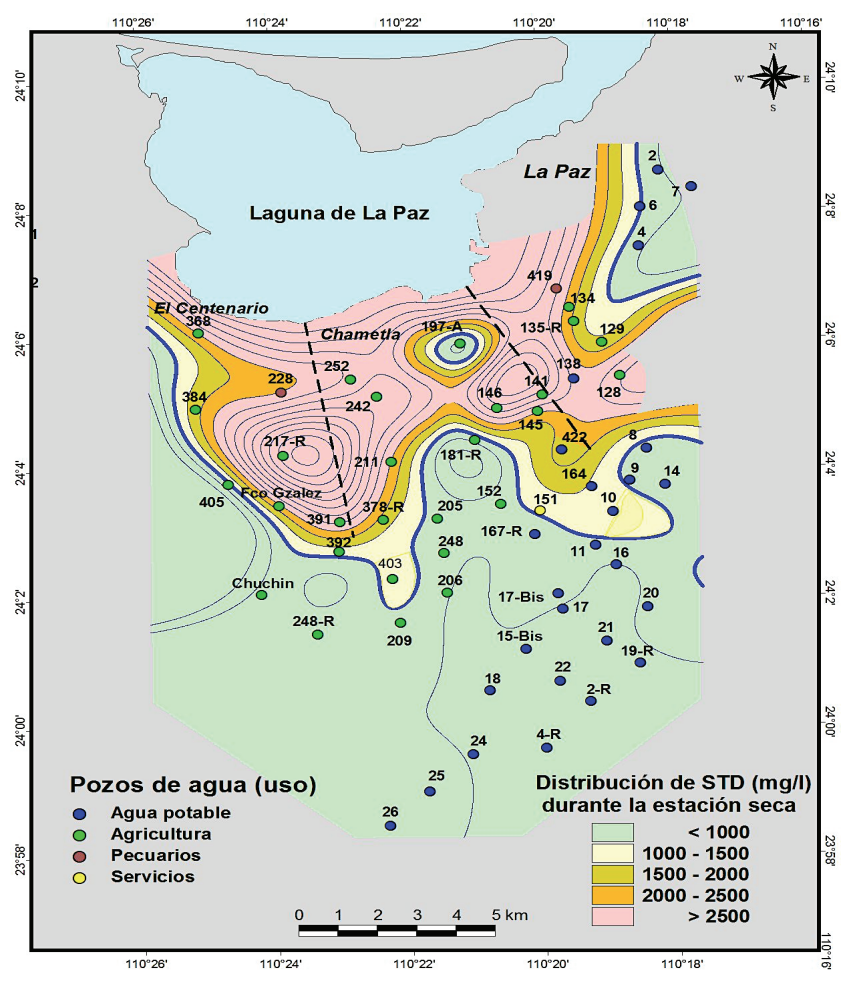

Figura 3a. Distribución de la concentración de STD en el acuífero de La Paz, durante la estación seca. La línea azul gruesa representa el límite de $1000 \mathrm{mg} \mathrm{L}^{-1}$, y las líneas rectas negras segmentadas indican el avance de la intrusión marina hasta el contorno de $2000 \mathrm{mg} \mathrm{L}^{-1}$.

encima de $1000 \mathrm{mg} \mathrm{L}^{-1}\left(2885,1120\right.$ y $2144 \mathrm{mg} \mathrm{L}^{-1}$, respectivamente). Indebidamente estos pozos son compartidos para riego agrícola y agua potable, por lo que están siendo sobreexplotados. El pozo 197-A es el único de los pozos agrícolas cercanos a la costa donde no se incrementó la concentración de STD (Figuras 3a y 3b), más bien disminuyó debido a un reducido bombeo.

El incremento en la concentración de STD en el agua subterránea de este acuífero costero no necesariamente es a causa de la intrusión marina. En años lluviosos el nivel del acuífero aumenta lo suficiente como para lavar la capa vadosa pudiendo capturar algunas sales. Este proceso parece que no ocurrió en el 2013 debido a la escasez de lluvias con $202.7 \mathrm{~mm}$ (promedio de 12 estaciones climatológicas de la CONAGUA) el cual estuvo por debajo de la media anual de $265 \mathrm{~mm}$ año-1 (CNA, 2005). Por otro lado, el agua de lluvia que se infiltra en las partes altas de la cuenca es transportada hacia el acuífero por flujo subterráneo, el cual lava las capas y rocas por donde fluye, transportando consigo iones y otros elementos hacia la zona costera alterando las concentraciones presentes.

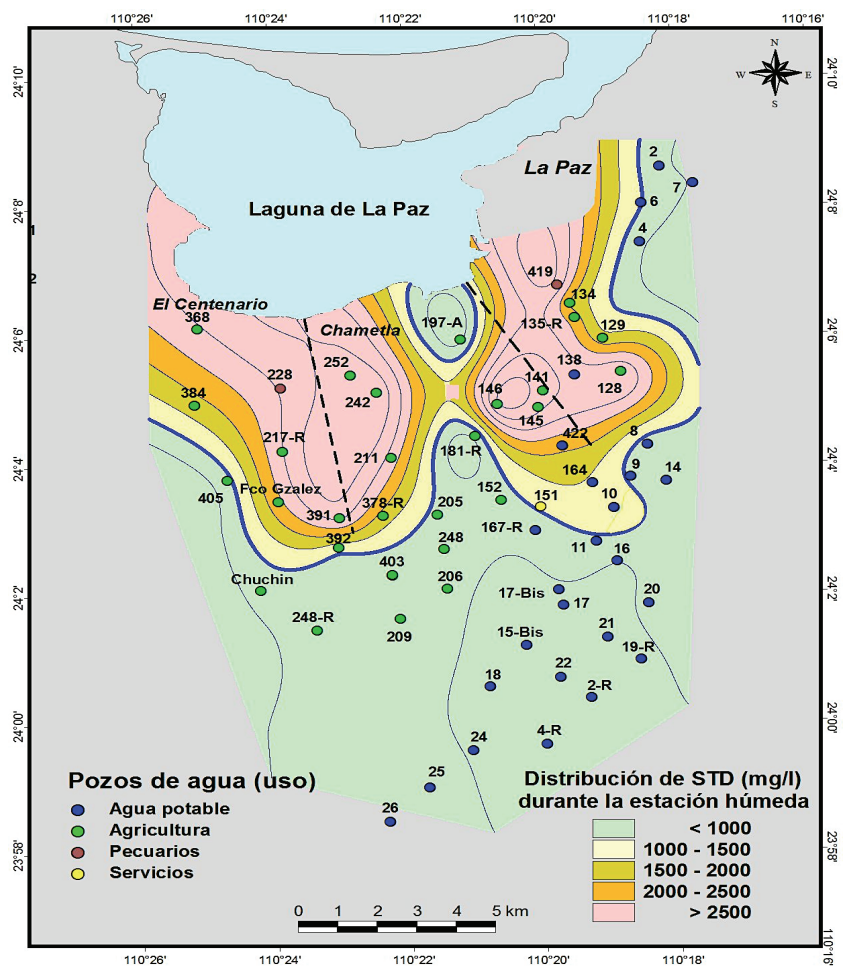

Figura 3b. Distribución de la concentración de STD en el acuífero de La Paz, durante la estación húmeda. La línea azul gruesa representa el límite de $1000 \mathrm{mg} \mathrm{L}^{-1}$, y las líneas rectas negras segmentadas indican el avance de la intrusión marina hasta el contorno de $2000 \mathrm{mg} \mathrm{L}^{-1}$.

\section{Distribución de Sales}

La Salinidad es la concentración total de sales disueltas en el agua, principalmente elementos como calcio, magnesio, sodio y potasio (cationes), y otros como carbonato, bicarbonato, sulfato y cloruro (aniones). El calcio se precipita fácilmente como carbonato de calcio $\left(\mathrm{CaCO}_{3}\right)$, que es el principal componente de la dureza del agua y causante de incrustaciones (USGS, 1999).

A partir de la distribución de concentración de STD en el agua subterránea se puede inferir y delimitar la intrusión marina en un acuífero costero, tal como lo hace la CONAGUA en los acuíferos de México, por lo que con medidas relativamente simples como el monitoreo de la conductividad eléctrica en los pozos de bombeo, se puede indicar la intrusión del agua de mar (Mahlknecht et al., 2017), no obstante, es importante considerar el tipo de sales presentes para verificar si corresponden al agua de mar. Al igual que las concentraciones de STD, la concentración de sales en el acuífero presentó una leve disminución después 
de las lluvias en la zona donde se localiza la batería de pozos de agua potable, sin embargo, cerca de la costa se observó un incremento.

Con los datos de CONAGUA de 14 pozos, se elaboró un diagrama de Piper (Figura 4) a partir de la relación entre los aniones (bicarbonatos, sulfatos y cloruros) y cationes principales (calcio, magnesio y sodio) (Freeze y Cherry, 1979).

A partir del gráfico de Piper se distinguieron cinco familias de agua (Figuras 4 y 5), donde destacan la clorurada-cálcica y clorurada-sódica. El anión predominante en concentración relativa fue el cloruro, presente en 12 pozos con un porcentaje promedio de 67\%; el bicarbonato con un 22\% dominó en solo dos pozos; y los sulfatos con el $11 \%$ son los que presentaron más baja concentración. El catión mayoritario en concentración relativa fue el calcio, con un porcentaje promedio de $43 \%$ predomina en nueve pozos; el sodio con $43 \%$ dominó en cinco pozos; y el magnesio con $14 \%$ es el que presentó concentraciones más bajas. Estos porcentajes reflejan un agua del tipo clorurada-cálcica en siete de los pozos $(10,138,145$, 243, 422, 378-R y 408), clorurada-sódica en cuatro de ellos (Ardilla 2, 134, 217-R y 368), clorurada cálcicasódica en el pozo 151, bicarbonatada-sódica en el pozo 197-A, y bicarbonatada cálcica-sódica en el pozo 6 (Figuras 4 y 5). Estos pozos en su mayoría agrícolas
(134, 145, 197-A, 217-R, 243, 368, 378-R y 408), de agua potable (Ardilla 2, 6 y 10), compartidos para riego agrícola y agua potable (138 y 422) y de servicios (151) presentaron alta concentración de STD (arriba de $1000 \mathrm{mg} \mathrm{L}^{-1}$ ), excepto el pozo agrícola 197-A con $483 \mathrm{mg} \mathrm{L}^{-1}$ (Cuadro 1) lo cual se atribuye a un limitado bombeo a pesar de encontrarse muy cerca del mar.

En estudios realizados por la CNA (1997) y CIGSA (2001) reportan que en el acuífero predominan las aguas de tipo clorurado-sódico, que atribuyen a la abundancia de sedimentos marinos en el subsuelo.

El agua subterránea contenida en los acuíferos es producto del agua de lluvia que se infiltra a través del suelo o de la roca, por lo que su composición química se altera por procesos geoquímicos de acuerdo a la constitución y contenido de minerales de las capas por donde circula (Custodio y Llamas, 2001), así la concentración de los diferentes iones puede incrementarse o disminuir.

Aunque el límite permisible de cloruros para agua potable en la NOM es de $250 \mathrm{mg} \mathrm{L}^{-1}$, en aguas subterráneas de zonas áridas son frecuentes las concentraciones superiores a los $1000 \mathrm{mg} \mathrm{L}^{-1}$ (Davis y Wiest, 1971). Los pozos 6, 151 y 197-A presentaron concentraciones menores a $250 \mathrm{mg} \mathrm{L}^{-1}$; los pozos Ardilla 2, 10, 134, 368 y 422 contienen menos de $1000 \mathrm{mg} \mathrm{L}^{-1} ; \mathrm{y}$ con concentraciones superiores a

Cuadro 1. Concentración de STD, aniones y cationes principales (unidades en $\mathrm{mg} \mathrm{L}^{-1}$ ) en agua de los pozos del acuífero de La Paz.

\begin{tabular}{lccccccccc}
\hline Pozo & Fecha & $\mathrm{Ca}^{2+}$ & $\mathrm{Mg}^{2+}$ & $\mathrm{Na}^{+}$ & $\mathrm{CO}_{3}^{2-}$ & $\mathrm{HHCO}_{3}{ }^{-}$ & $\mathrm{SO}_{4}^{2-}$ & $\mathrm{Cl}^{-}$ & $\mathrm{STD}^{2-}$ \\
\hline Ardilla 2 & $6 / 5 / 2008$ & 109.00 & 54.00 & 272.00 & 20.00 & 235.00 & 89.00 & 479.00 & 1037.00 \\
6 & $4 / 15 / 2013$ & 127.55 & 39.92 & 125.25 & 5.00 & 107.00 & 10.48 & 82.97 & 1026.00 \\
10 & $4 / 15 / 2013$ & 211.99 & 79.03 & 64.18 & 5.00 & 159.00 & 93.38 & 524.84 & 1403.00 \\
134 & $4 / 15 / 2009$ & 153.31 & 50.63 & 452.31 & 5.00 & 244.65 & 142.50 & 799.75 & 1918.00 \\
138 & $4 / 15 / 2013$ & 368.90 & 128.15 & 259.93 & 5.00 & 207.00 & 303.76 & 1155.89 & 2885.00 \\
145 & $4 / 29 / 2013$ & 296.70 & 91.37 & 186.40 & 5.00 & 160.06 & 154.99 & 1043.51 & 2576.00 \\
151 & $4 / 08 / 2008$ & 104.93 & 39.23 & 99.23 & 5.00 & 144.00 & 31.00 & 221.21 & 1077.00 \\
$197-\mathrm{A}$ & $4 / 29 / 2013$ & 34.30 & 15.00 & 68.02 & 5.00 & 197.16 & 30.99 & 101.08 & 484.00 \\
$217-\mathrm{R}$ & $12 / 16 / 2008$ & 490.35 & 178.22 & 685.47 & 5.00 & 200.00 & 204.00 & 1987.56 & 6204.00 \\
243 & $4 / 15 / 2009$ & 545.09 & 146.40 & 317.42 & 5.00 & 166.95 & 170.00 & 1659.49 & 3398.00 \\
368 & $4 / 08 / 2008$ & 326.00 & 84.41 & 472.76 & 5.00 & 218.00 & 242.50 & 907.52 & 2519.00 \\
$378-\mathrm{R}$ & $4 / 29 / 2013$ & 197.50 & 49.45 & 132.50 & 5.00 & 164.30 & 65.19 & 1094.98 & 1778.00 \\
408 & $4 / 08 / 2008$ & 674.55 & 237.27 & 573.42 & 5.00 & 180.00 & 174.00 & 1642.51 & 5988.00 \\
422 & $4 / 15 / 2013$ & 275.11 & 91.35 & 209.81 & 5.00 & 144.00 & 143.36 & 914.72 & 2144.00 \\
\hline
\end{tabular}

Pozos monitoreados y analizados por la CONAGUA (2008, 2009 y 2013). 


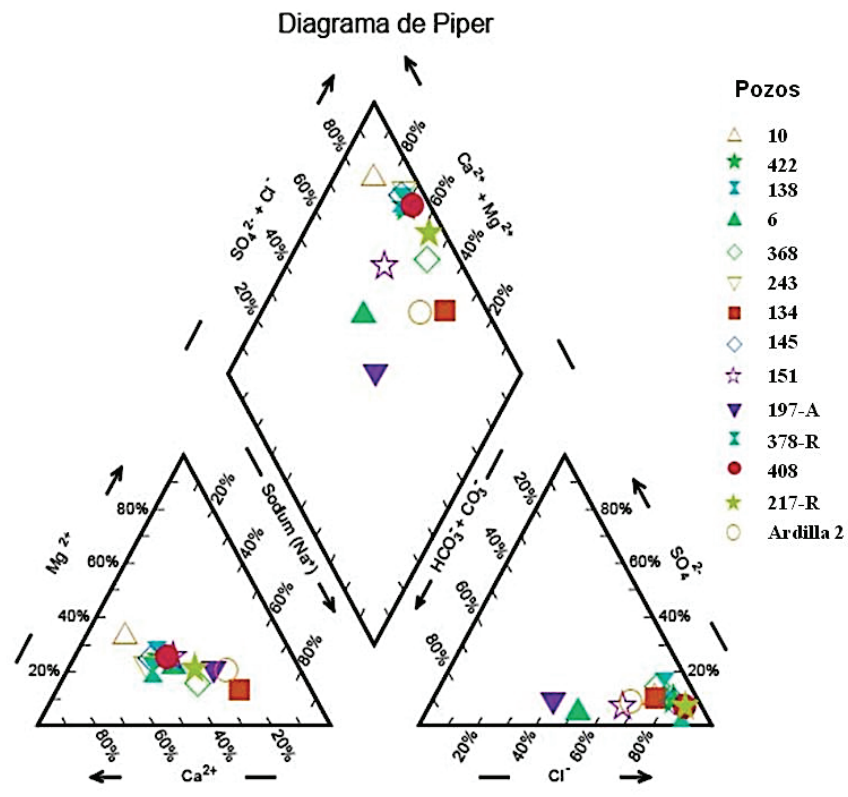

Figura 4. Diagrama de Piper para la determinación de las familias de agua de 14 pozos. Datos de la CONAGUA (2008, 2009 y 2013).

los $1000 \mathrm{mg} \mathrm{L}^{-1}$ están los pozos 138, 145, 217-R, 243, 378-R y 408. Esta concentración alta de cloruros supone un tiempo largo de permanencia del agua dentro del acuífero. Por ejemplo, en los pozos 138, 217-R, 243, 368 y 422, se cumple la relación $\mathrm{Cl}>$ $\mathrm{SO}_{4}>\mathrm{HCO}_{3}$ (Custodio y Llamas, 2001). Estos pozos cercanos a la línea de costa presentan evidencia de intrusión marina debido a la alta concentración de STD (Cuadro 1) mayor a $2000 \mathrm{mg} \mathrm{L}^{-1}$, pero además por su elevada concentración de cloruros se confirma que la alta salinidad proviene del mar, más que por disolución de minerales que conforman los materiales del acuífero o por contaminación antropogénica. Tamez Meléndez et al. (2016) reportan que debido a los altos volúmenes de extracción de agua que se llevan a cabo para riego agrícola, el nivel freático se ha modificado a lo largo de la costa a causa de la intrusión marina, lo que ha provocado la inversión del flujo subterráneo en esta zona. Es así que las concentraciones de STD y cloruros se han incrementado dramáticamente.

En los pozos cercanos a la costa, tomando en cuenta que el ion cloro es el principal soluto en el agua de mar, es evidente que una alta concentración de cloruros sea atribuida a la intrusión marina. Pero en pozos más alejados de la costa, la fuente de cloruros puede proceder de depósitos sedimentarios por donde circula el agua subterránea, en este caso, puede provenir de sedimentos marinos que conforman la estratigrafía del acuífero, así

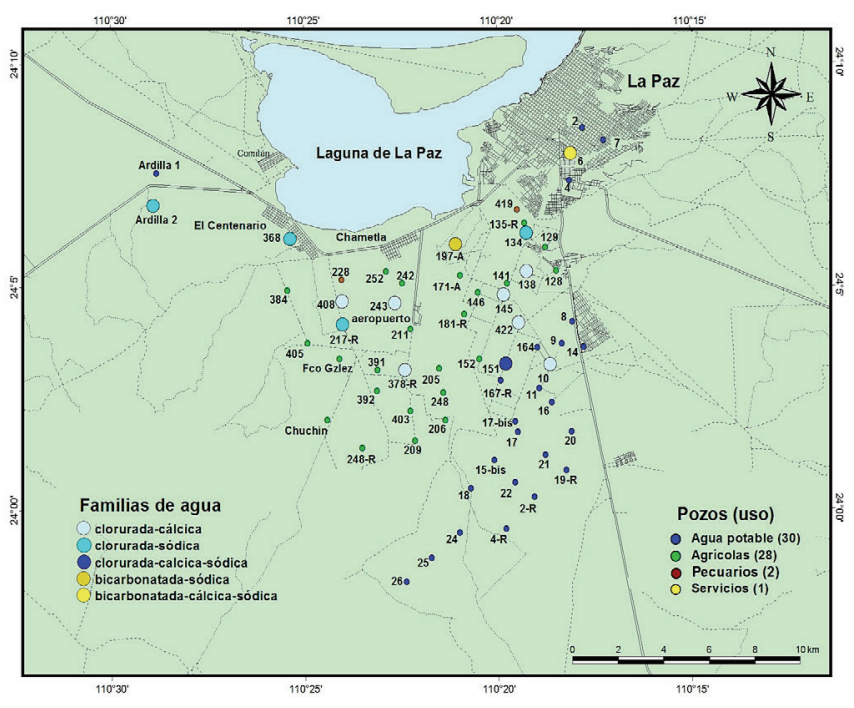

Figura 5. Familias de agua de 14 pozos del acuífero de La Paz. Se incluyen los pozos 243 y 408 que no están considerados en la Red de Monitoreo (Figura 2).

como de la disolución de $\mathrm{NaCl}$ y minerales afines que existen en las formaciones de evaporitas. Otra posible procedencia de los cloruros puede ser de los minerales como la sodalita y la apatita que componen las rocas ígneas y metamórficas características de la parte alta de la cuenca de La Paz, que contienen cloruros como constituyente fundamental, pero también las micas, hornblendas y vidrios volcánicos, sin embargo, es poca la posibilidad de que los cloruros provengan de estas fuentes debido a que tienen baja solubilidad (Davis y Wiest, 1971). Fetter (2001; en Rosales Ramírez, 2012) ${ }^{4}$ reporta que el ion cloro es un elemento poco común en las rocas de los acuíferos, por lo que es mayor la posibilidad de que los cloruros provengan del agua de mar. Debido a la alta solubilidad de las sales, los patrones de flujo y tiempos de residencia rápidamente pasan a la fase acuosa pudiendo alcanzar concentraciones muy altas.

Aunque la NOM no exhibe un límite permisible para el calcio en el agua potable, en otros países está limitado a $250 \mathrm{mg} \mathrm{L}^{-1}$. La concentración de calcio en los pozos 138, 145, 217-R, 243, 368, 408 y 422, fue por arriba del límite permisible (Cuadro 1). La concentración de sodio en los pozos Ardilla 2, 134, 138, 217-R, 243, 368, 408 y 422, fue superior a los $200 \mathrm{mg} \mathrm{L}^{-1}$ que es límite permisible establecido en la NOM. Así mismo, la concentración de magnesio en los pozos Ardilla 2, 10, 138, 145, 217-R, 243, 368, 408 y 422 (Cuadro 1) fue por arriba del límite establecido en la NOM. 
Las sales disueltas en el océano están formadas por diez elementos principales, el ion cloro es el principal soluto en la composición del agua de mar y las sales de sodio y de magnesio $\left(\mathrm{NaCl}\right.$ y $\left.\mathrm{MgCl}_{2}\right)$ son las más abundantes (Davis y Wiest, 1971). De lo anterior se deduce que la alta concentración de los iones cloro, sodio y magnesio (Cuadro 1) en los pozos que se encuentran relativamente cercanos al mar son aportados por sales provenientes de la intrusión marina.

La alta concentración de los iones cloro y calcio (Cuadro 1) sugiere que algunos pozos reciben aportación de calcio por la lixiviación de materiales que componen la estratigrafía del acuífero, que son lavados por los flujos de agua subterránea. El calcio en rocas sedimentarias aparece como $\mathrm{CaCo}_{3}$ (calcita y aragonito) y $\mathrm{CaMg}\left(\mathrm{CO}_{3}\right)_{2}$ (dolomita), y en las rocas ígneas se encuentra en las plagioclasas cálcicas $\left(\mathrm{Ca}(\mathrm{Si}, \mathrm{Al})_{3} \mathrm{O}_{8}\right)$ (Rosales Ramírez, 2012) ${ }^{4}$. Las áreas de recarga del acuífero de La Paz se localizan hacia el este y sureste en las partes altas de la cuenca de La Paz (SARH-UNAM-UABCS, 1986; CIGSA, 2001; IPNCICIMAR/CIBNOR/UABCS, 2002) donde el agua de lluvia captada, se infiltra y transporta lentamente por flujo subterráneo hacia las partes bajas de la cuenca para recargar el acuífero (Cruz Falcón et al., 2011). A través de este proceso el agua circula entre algunas capas de arenisca-conglomerado intercalada con limolitas, arcillas y horizontes fosilíferos cementados por $\mathrm{CaCO}_{3}$ (SARH-UNAM-UABCS, 1986; Álvarez Arellano et al., 1997; CNA, 1997; CIGSA, 2001; IPN-CICIMAR/CIBNOR/UABCS, 2002), arrastrando consigo estos elementos. Además, existen capas o depósitos de caliche mezclados con materiales aluviales que afloran en algunas áreas de la cuenca de La Paz (Nava Sánchez) 5 .

Lo anterior plantea una amplia zona de mezcla de agua de mar debido a la intrusión marina con agua dulce producto de la recarga natural por flujo subterráneo que alcanza a llegar a los pozos en la zona costera. Mahlknecht et al. (2017) reportan que se está produciendo un importante proceso de intercambio de cationes en el acuífero, con sodio de agua de mar reemplazando el calcio de procedencia continental. Además, el agua de mar con $\mathrm{Na}^{+}$y $\mathrm{Cl}^{-}$como iones dominantes está en contacto con los sedimentos del área costera y su intrusión induce un intercambio de cationes en las partículas del suelo donde se absorbe el $\mathrm{Na}^{+}$y se libera $\mathrm{Ca}^{2+}$ (Tamez Meléndez et al., 2016). En cualquier caso, para determinar si el proceso de intrusión marina prevalece y se extiende, o se encuentra estabilizado, es necesario utilizar algún otro método, o llevar a cabo una relación iónica, pero desafortunadamente en este estudio no se cuenta con datos suficientes.

Con base en las familias de agua determinadas y la distribución de la concentración deSTD, seidentificaron tres zonas principales del acuífero de La Paz (Figura 6): Zona 1, delimitada por dos áreas cercanas a la costa bajo la influencia de la intrusión marina y concentraciones de STD mayores a $2000 \mathrm{mg} \mathrm{L}^{-1}$, las cuales pertenecen a las familias clorurada-cálcica y clorurada-sódica; Zona $2 \mathrm{a}$, representada por un área pequeña cercana a la costa, con baja la influencia de intrusión marina y baja concentración de STD (500 a $1000 \mathrm{mg} \mathrm{L}^{-1}$ ) que pertenece a la familia bicarbonatada-sódica; Zona $2 \mathrm{~b}$, alejada de la línea de costa, con baja influencia de intrusión marina y baja concentración de STD (500 a $\left.1000 \mathrm{mg} \mathrm{L}^{-1}\right)$ que pertenece a la familia clorurada y bicarbonatada cálcica-sódica; Zona 2c, relativamente alejada de la línea de costa, con mediana influencia de intrusión marina y moderada concentración de STD (900 a $1800 \mathrm{mg} \mathrm{L}^{-1}$ ) que pertenece a la familia cloruradasódica; y la Zona 3, con concentración de STD de 300 a $1000 \mathrm{mg} \mathrm{L}^{-1}$, la cual no cuenta con información de aniones y cationes, por lo que se desconoce el tipo

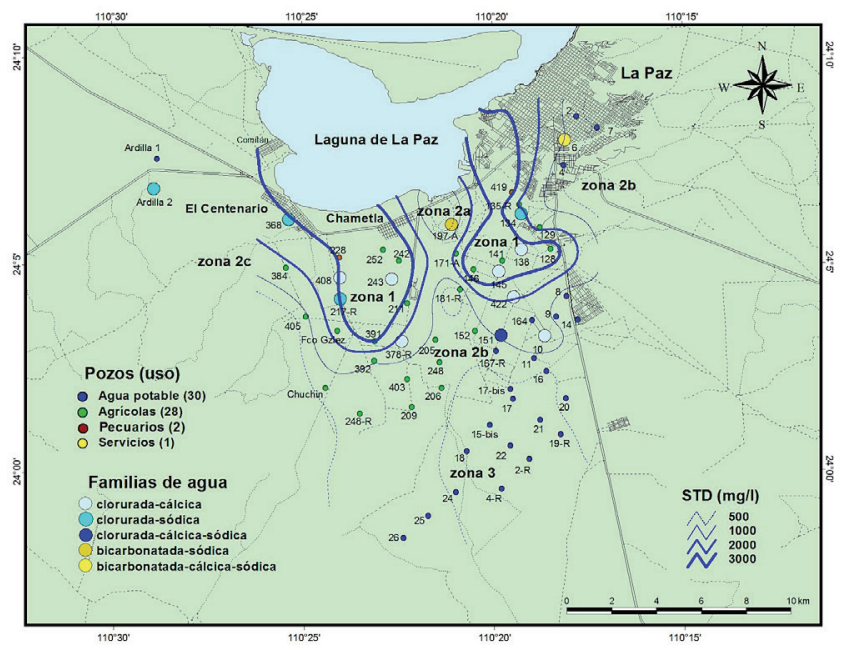

Figura 6. Zonas del acuífero de La Paz, identificadas por la concentración de STD y familias de agua.

\footnotetext{
${ }^{4}$ Rosales Ramírez, T. Y. 2012. Reconocimiento químico e isotópico del acuífero costero de La Paz, B.C.S: Evaluación de una posible intrusión marina. Tesis de Maestría, CICESE. Ensenada, Baja California, México. 123 p.

${ }^{5}$ Nava Sánchez, E. H. com. pers. Investigador Titular, Departamento de Geología Ambiental del Centro Interdisciplinario de Ciencias Marinas (CICIMAR-IPN), La Paz, BCS, México.
} 
de agua, pero se infiere que pertenece a la familia bicarbonatada-cálcica por estar lejos de la influencia de la intrusión marina y donde sus características derivan de la lixiviación de materiales propios del acuífero. La familia de agua bicarbonatada es característica de agua de reciente infiltración y por tanto con trayectoria de flujos relativamente cortos (Freeze y Cherry, 1979), por lo que es factible encontrarla en la parte interna del acuífero o las áreas cercanas a las zonas de recarga.

\section{CONCLUSIONES}

- El acuífero de La Paz presenta problemas de intrusión marina en el frente costero, causados por la sobreexplotación, lo cual se infiere por la alta concentración de STD, así como por el alto contenido de cloruros, sodio y magnesio en algunos pozos cercanos a la costa.

- La mayoría de los pozos agrícolas tienen una alta concentración de STD, mientras que casi todos los pozos de agua potable, que en su mayoría se localizan en la parte central-sur del acuífero, están por abajo del límite permisible de la Norma Oficial Mexicana.

- De los 61 pozos muestreados 27 resultaron con una concentración de STD superior a los $1000 \mathrm{mg} \mathrm{L}^{-1}$. De estos, 18 pozos son agrícolas; 6 pozos de agua potable (dos son de uso compartido para riego agrícola-agua potable); dos pecuarios y uno de servicios. Por lo que el sector agrícola y pecuario es el que tiene mayores problemas con el incremento de sales a causa de la sobreexplotación.

- Se considera que las altas concentraciones de cloruros, sodio y magnesio, encontradas en algunos pozos cercanos a la costa se deben a la intrusión marina. Mientras que el calcio, también presente en concentraciones elevadas, es lixiviado de las rocas que forman la estratigrafía interior del acuífero.

- Se distinguieron cinco familias de agua de los datos analizados, de los que predominan la clorurada-cálcica y la clorurada-sódica, lo cual plantea que existe una amplia zona de mezcla de agua de mar con agua dulce producto de la recarga natural.

- Se identificaron tres zonas principales del acuífero con base en las familias de agua y distribución de STD encontrados, en que resalta la zona central-sur donde se ubica la batería de pozos de agua potable que abastecen a la ciudad de La Paz, la cual presenta baja influencia de intrusión marina y baja concentración de STD.
- Para controlar la sobreexplotación y la intrusión marina al acuífero, es necesario implementar lo antes posible programa de manejo integral del mismo por las autoridades correspondientes para dar solución o evitar que se incremente este problema. Se recomienda medir y controlar los volúmenes de agua que se extraen de los pozos para reducir el avance de la de intrusión marina, así como un monitoreo continuo de los pozos para obtener una evaluación adecuada en la evolución de la concentración de STD y sales.

\section{AGRADECIMIENTOS}

A la Sociedad de Historia Natural Niparajá, por el apoyo a través del convenio institucional con CIBNOR, proyecto: Niparajá - Monitoreo de Pozos del acuífero de La Paz (2013). Al M.C. Alvaro González Michel por su apoyo en el trabajo de campo para la medición de parámetros físico-químicos, toma de muestras de agua de pozo; a Juan E. Vega Mayagoitia por su apoyo en la logística y trabajo de campo; René Gaxiola Márquez y Francisco Aguillón León, por su ayuda en pruebas de campo. A la M.C. Karen Velázquez, estudiante de Doctorado de CICIMAR-IPN, por la elaboración del diagrama de Piper.

\section{LITERATURA CITADA}

Álvarez Arellano, A. D., H. Rojas S. y J. J. Prieto M. 1997. Geología de la Bahía de La Paz y áreas adyacentes. pp. 13-29. In: J. Urbán R. y M. Ramirez R. (eds.). La Bahía de La Paz, investigación y conservación. UABCS-CICIMAR-SCRIPPS. La Paz, Baja California Sur, México. ISBN: 968-896-083-7.

Carrillo Chávez, A., J. I. Drever, and M. Martínez. 2000. Arsenic content and groundwater geochemistry of San Antonio-El Triunfo, Carrizal and Los Planes aquifers in southernmost Baja California, México. Environ. Geol. 39: 1295-1303.

CCA, CONAGUA, FMEA (Consejo Consultivo del Agua A.C., Comisión Nacional del Agua, Fundación Mexicana para la Educación Ambiental, A.C.). 2005. Documento base para discusión caso de estudio manejo sustentable del agua en la región de La Paz BCS. 54 p.

CIGSA (Consultores en Ingeniería Geofísica S. A. de C. V.). 2001. Estudio de caracterización y modelación de la intrusión marina en el acuífero de La Paz B. C. S. Contrato CNA, GAS-013PR01. 284 p.

CNA (Comisión Nacional del Agua). 1997. Censo de Captaciones de Aguas Subterráneas y Colección de datos Geohidrológicos en la zona La Paz-El Carrizal, B.C.S. Informe final. Estudio realizado por ADI CONSTRUCCIONES, S.A. DE C.V., Contrato No. GAS-026-PR097. Subdirección General Técnica, Gerencia de Aguas Subterráneas. 173 p. México, D. F. 
CNA (Comisión Nacional del Agua). 2005. Estudio para actualizar la disponibilidad media anual de las aguas nacionales superficiales en las 85 (ochenta y cinco) subregiones hidrológicas de las 7 (siete) regiones hidrológicas 1,2,3,4,5,6 y 7 de la Península de Baja California, mediante la aplicación de la NOM-011-CNA-2000. México, D. F.

CONAGUA (Comisión Nacional del Agua). 2009. Actualización de la disponibilidad media anual de agua subterránea, acuífero (0324) La Paz, estado de Baja California Sur. Publicada en el Diario Oficial de la Federación el 28 de Agosto de 2009. 17 p. México, D. F.

CONAGUA (Comisión Nacional del Agua). 2010. Manejo integrado de las aguas subterráneas en el acuífero La Paz, Baja California Sur. Proceso de planeación. Organismo de cuenca península de Baja California, Dirección local Baja California Sur, Subdirección de asistencia técnica operativa. La Paz, BCS. Tomo I, 211 p. México, D. F.

Cruz Falcón, A., R. Vázquez González, J. Ramírez Hernández, E. H. Nava Sánchez, E. Troyo Diéguez, J. Rivera Rosas y J. Vega Mayagoitia. 2011. Precipitación y recarga en la cuenca de La Paz, BCS, México. Rev. Univ. Cienc. 27: 250-263.

Cruz Falcón, A., J. Ramírez Hernández, R. Vázquez González, E. H Nava Sánchez, E. Troyo Diéguez y H. C. Fraga Palomino. 2013. Estimación de la recarga y balance hidrológico del acuífero de La Paz, BCS, México. Rev. Univ. Cienc. 29: 87-100.

Custodio, E. y M. R. Llamas. 2001. Hidrología subterránea. Omega. Barselona, España. ISBN 13: 9788428204477.

Davis, S. N. and R. De Wiest. 1971. Hidrogeología. Ariel. Barcelona, España. ASIN: B00OF6KRTY.

Dragoo, L. C. and T. Maddock 2004. Hydrology model for the alternative futures of La Paz. Harvard University, Universidad Autónoma de Baja California Sur and the University of Arizona, Department of Hydrology and Water Resources. Tucson, AZ, USA.

Escolero, O. y S. Torres Onofre. 2007. Análisis de la intrusión de agua de mar en el acuífero de La Paz (México). Bol. Geol. Minero 118: 637-648.
ESRI (Environmental Systems Research Institute). 2009. ArcGIS Versión 9.3.1. 380. http://www.esri.com. Redlands, CA, USA.

Freeze, A. R. and J. A. Cherry. 1979. Groundwater. Prentice-Hall. Upper Saddle River, NJ, USA.

IPN-CICIMAR/CIBNOR/UABCS (Instituto Politécnico Nacional, Centro Interdisciplinario de Ciencias Marinas, Centro de Investigaciones Biológicas del Noroeste, Universidad Autónoma de Baja California Sur). 2002. Ordenamiento Ecológico Bahía de La Paz, B.C.S. Informe preliminar. Abril, 2002. $466 \mathrm{p}$

Mahlknecht, J., D. Merchán, M. Rosner, A. Meixner, and R. Ledesma Ruiz. 2017. Assessing seawater intrusion in an arid coastal aquifer under high anthropogenic influence using major constituents, $\mathrm{Sr}$ and $\mathrm{B}$ isotopes in groundwater. Sci. Total Environ. 587-588: 282-295. http://doi.org/10.1016/j. scitotenv.2017.02.137.

Norma Oficial Mexicana NOM-127-SSA1-1994, Salud ambiental. 1994. Agua para uso y consumo humano. Límites permisibles de calidad y tratamientos a que debe someterse el agua para su potabilización. Diario Oficial de la Federación. México, D. F.

SARH-UNAM-UABCS (Secretaría de Agricultura y Recursos Hidráulicos, Universidad Nacional Autónoma de México, Universidad Autónoma de Baja California Sur). 1986. Estudio Geohidrológico complementario de las cuencas La Paz-El Carrizal, para proporcionar agua en bloque a la ciudad de La Paz, Baja California Sur. 334 p.

Steinitz, C., R. F. M. Flaxman, J. C. Vargas-Moreno, T. Canfield, O. Arizpe, M. Ángeles, M. Cariño, F. Santiago, T. Maddock III, C. Dragoo, K. Baird, and L. Godínez. 2006. Alternative futures for the region of La Paz, Baja California Sur, Mexico. Harvard University. Cambridge, MA, USA.

Tamez Meléndez, C., A. Hernández Antonio, P. C. Gaona Zanella, N. Ornelas Soto, and J. Mahlknecht. 2016. Isotope signatures and hydrochemistry as tools in assessing groundwater occurrence and dynamics in a coastal arid aquifer. Environ. Earth Sci. 75:830. http://dx.doi.org/10.1007/s12665-016-5617-2.

USGS (United States Geological Survey). 1999. Ground Water. U.S. Geological Survey General Interest Publication. Quality of Ground Water. Reston, Virginia, revision 1999. http://pubs. usgs.gov/gip/gw/index.html. (Consulta: marzo 21, 2017). 